\title{
Hard Dead Zone and Friction Modeling and Identification of a Permanent Magnet DC Motor Non-Linear Model
}

\author{
C.A PEREZ-GOMEZ \\ MCIE \\ Universidad Autónoma \\ Metropolitana-Azcapotzalco \\ CDMX \\ MEXICO
}

\author{
J.U. LICEAGA-CASTRO \\ Departamento de Electrónica \\ Universidad Autónoma \\ Metropolitana-Azcapotzalco \\ CDMX \\ MEXICO
}

\author{
I.I. SILLER-ALCALA \\ Departamento de Electrónica \\ Universidad Autónoma \\ Metropolitana-Azcapotzalco \\ CDMX \\ MEXICO
}

\begin{abstract}
The identification of a permanent magnet DC motor model including non-linearities dead zone, Coulomb friction, and viscous friction, is presented. The dead zone considered here is the so call "hard" dead zone, whereas the friction force is modeled in two different ways: first, considering the value of viscous coefficient friction as a constant and second, approximating viscous coefficient by a polynomial depending on motors rotor velocity. The polynomial representation of the viscous friction value allows it to be adjusted automatically as a function of the speed of the system, as occurs in real systems. Therefore, a model capable of better representing the real motor behavior along a wide range of operation is obtained. The non-linear model is validated and compared using real-time data obtained from Quanser's direct current motor control trainer system, using the numerical tool Matlab ${ }^{\circledR} /$ Simulink $^{\mathrm{TM}}$.
\end{abstract}

Keywords: - Non-linear Model, Dead zone, Coulomb Friction, Viscous Friction, DC motor.

Received: May 2, 2020. Revised: September 5, 2020. Accepted: September 18, 2020. Published: October 7, 2020.

\section{Introduction}

The most common type of actuator in electromechanical systems is the direct current (DC) motor. The permanent magnet DC motor is the simplest type of these devices. They have been extensively used due to their small physical size, relatively low cost, and high efficiency in a wide variety of applications such as: windshield wipers, personal computer fans or as electromagnetic actuators in biomedical equipment, robotic manipulators and many other industrial applications, [1]-[3].

The simplicity of its construction makes the permanent magnet DC (PMDC) motor relatively easy to understand and control. These motors do not have field winding; therefore, the control is through the armature winding. The general control methods for these devices are based on the classic PID controllers, modern and intelligent controllers. [4], [5].

Usually, this type of motors is modeled as second order linear system neglecting or disregarding the non-linearities present in the system like the dead zone and Coulomb friction, [6], [7]. Neglecting or ignoring these non-linearities can affect the performance of control systems, especially in those cases where the control system is designed to operate at low speeds or for position control objectives. That is, in the case of speed control systems this simplification does not affects its performance as these non-linearities acts at very low or zero speed. However, for position control purposes, where the motor operates around zero speed, these nonlinearities induce oscillations in the motor response, avoiding the possibility of achieving zero steady state error. Moreover, including these non-linearities becomes a must when high precision is required specially under variable loads, [8]- [10]. Obtaining good models for electromechanical systems, including DC motors, involves modeling non-linearities such as dead zone and friction. Also, not only the modeling but the identification of these non-linearities is not trivial, so building an accurate model is a difficult task, [11], [12]. Different strategies had been applied to estimate motors parameters based on transient responses analysis as those reported in $[13,14]$. Nonetheless, in this paper the Strejc method is adopted because its simplicity for the identification of first order systems and, also because the model is subdivided into two first order systems.

However, in the case of speed control, where viscous friction coefficient is assumed constant, it is 
well known that it depends on the velocity affecting the constant time and steady state gain of linear motor models. Therefore, modeling viscous friction for a long range of speeds is also required for speed control purposes.

In recent years, new approaches have been developed to obtain more precise models better representing the behavior of systems such as electric motors. Also, by having better and more accurate models, the validation and testing of different control approaches through digital simulations allows reducing real time implementation problems in the actual process, [15]. In this paper a validate PMDC motor non-linear model is obtained. The model includes dead zone and friction, whose parameters are identified by experimental tests. The selected PMDC motor is the Maxon motor of the Quanser DC Motor Control Trainer (DCMCT) system. The dead zone is modeled by the "hard dead zone" model and the friction model used is the Coulomb plus viscous friction model. Likewise, a new approach to the viscous friction model is obtained through a polynomial fit, to get a model of the PMDC motor capable of representing the real system in a wider range of speeds.

The main objective of this paper is to provide a validated nonlinear model for a PMDC motor which includes the most important nonlinearities for position and speed control purposes with a methodology for its identification.

The paper is structure as follows. Section 2 presents the model for the PMDC motor and the non-linearities. Section 3 explains the system's parameters estimation. Section 4 describes the design model in Matlab ${ }^{\circledR} /$ Simulink $^{\mathrm{TM}}$ including the dead zone and Coulomb plus viscous friction. The model is validated in Section 5, the polynomial approximation of friction is obtained in Section 6 and, finally in Section 7 conclusions are presented.

\section{Mathematical Modeling}

\subsection{PMDC Motor Model}

The PMDC motor can be modeled using two linear equations for the electrical and mechanical subsystems.

$$
\begin{gathered}
v(t)=R i(t)+L \frac{d i(t)}{d t}+E_{a} \\
T_{m}(t)=k_{m} i(t)=J \frac{d^{2} \theta(t)}{d t^{2}}+b \frac{d \theta}{d t}
\end{gathered}
$$

Equation (1) represents the electrical subsystem where $v(t)$ is the applied armature voltage, $i(t)$ is the armature current, $E_{a}=k_{m} \frac{d}{d t} \theta(t) \quad$ counter electromotive force and, $L$ and $R$ represent the inductance and resistance of armature winding, respectively. In this model the counter electromotive force present in the motor is assumed negligible because the motor is operating at very low speed, that is, $\frac{d}{d t} \theta(t) \approx 0$.

Expression (2) represents the mechanical subsystem where $T_{m}(t)$ is the magnetic torque, $k_{m}$ is the motor constant, $J$ is the motors rotor equivalent moment of inertia, $b$ is the friction coefficient and $\theta$ is the angular position of the rotor.

Transfer function $G_{e}(s)$ of the electrical subsystem results in:

$$
G_{e}(s)=\frac{I(s)}{V(s)}=\frac{\frac{1}{R}}{\left(\frac{L}{R} s+1\right)}=\frac{K_{e}}{\left(\tau_{e} s+1\right)}
$$

Where the steady state gain $K_{e}$ of the electrical subsystem is given by $1 / R$ and the time constant $\tau_{e}=L / R$.

Expression (4) shows the transfer function for the mechanical subsystem with $\omega(t)=\dot{\theta}$ as the rotor velocity.

$$
\begin{gathered}
G_{m}(s)=\frac{\omega(s)}{T_{m}(s)}=\frac{\frac{1}{b}}{\left(\frac{J}{b} s+1\right)} ; \\
T_{m}(s)=k_{m} I(s)
\end{gathered}
$$

Therefore, the transfer function relating rotors velocity $\omega(t)$ to input voltaje $v(t)$ is given by:

$$
\frac{\omega(s)}{V(s)}=\frac{\frac{k_{m}}{R b}}{\left(\frac{J}{b} s+1\right)\left(\frac{L}{R} s+1\right)}
$$

Transfer function (5) can be simplified by pole dominance because the electrical mode is faster than the mechanical mode, [7], [16]; thus, the PMDC motor transfer function reduces to:

$$
\frac{\omega(s)}{V(s)}=\frac{\frac{k_{m}}{R b}}{\left(\frac{J}{b} s+1\right)}=\frac{K_{m o t}}{\tau_{m o t} s+1}
$$

where the steady state gain of the PMDC motor $K_{m o t}$ is $k_{m} / R b$ and the time constant is $\tau_{m o t}=\mathrm{J} /$ $b$. 
From (3) and (4) is clear that is possible to identify mechanical and electrical subsystems transfer functions by the analysis of their step responses.

\subsection{Dead Zone Model}

Commonly, the non-linear dead zone is represented by the "smooth" dead zone shown in the figure 1,

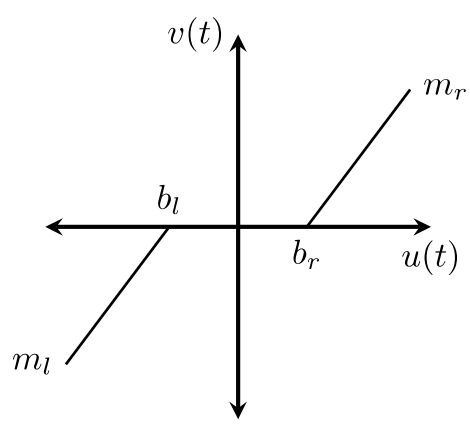

Fig. 1. "Smooth" dead zone.

where $u(t)$ is the input, $v(t)$ is the output, $b_{l}$ and $b_{r}$ are the left and right break points while $m_{l}$ and $m_{r}$ are the slopes of the dead zone, respectively. When the dead zone is symmetric $b_{l}=b_{r}$ and $m_{l}=m_{r}$, [15], [16]. Nevertheless, this approximation does not necessarily accurately represent the real physical phenomenon in the case of electric motors. That is, even with a small input voltage the electrical subsystem can be active but inducing a magnetic torque no capable of breaking inertia and friction in the mechanical subsystem. Therefore, in the case of electric motors dead zone is located between electrical and mechanical subsystems.

For this reason, the dead zone is modeled as shown in figure 2, [19] this representation is called the "hard dead zone" which is considered a better approximation of the non-linear phenomenon that occurred in the PMDC motor.

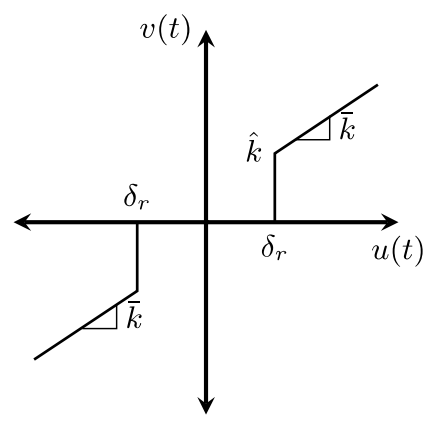

The symmetric "hard dead zone" is represented as shown in (7)

$$
v(t)=\left\{\begin{aligned}
\operatorname{sign}(u(t))[\bar{k}|u(t)|+\hat{k}] & ;|u(t)| \geq \delta_{r} \\
0 \quad & ;|u(t)|<\delta_{r}
\end{aligned}\right.
$$

where $u(t)$ is the input of the system, $v(t)$ is the output, $\delta_{r}$ represents the break point of the dead zone, $\hat{k}$ represents the sudden offset of the system by breaking inertias and $\bar{k}$ is the slope of the dead zone. The dead zone is normally assumed to be a phenomenon at the process input signal. However, this is not true in the case of electric motors. In fact, the electrical subsystem can be active even with a minimal input voltage signal that unfortunately generates a magnetic torque that is not capable of inducing movement to the rotor. Therefore, the dead zone is a phenomenon occurring between the electrical subsystem and the mechanical subsystem

\subsection{Friction Model}

The friction can be defined as the tangential reaction force between two surfaces in contact. There are several models that represent the phenomenon, the most used is the Coulomb plus viscous friction model as shown in figure 3. [20, 22].

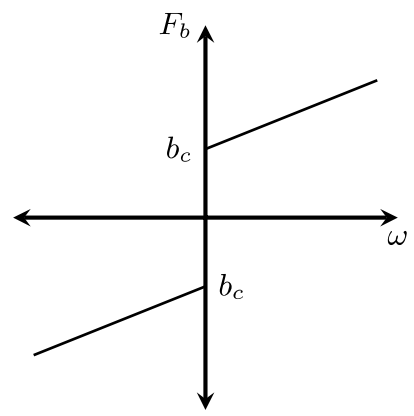

Fig. 3. Coulomb plus viscous friction model.

This model is described by expression (8)

$$
F_{b}=\left\{\begin{array}{l}
b_{c} \cdot \operatorname{sign}(\omega)+b_{v} \omega ; \omega \neq 0 \\
F_{a p} ; \omega=0 \text { and } F_{a p}<b_{c}
\end{array}\right.
$$

where $F_{b}$ is the friction force, $b_{c}$ is the Coulomb friction force, $\omega$ is the speed, $b_{v}$ is the viscous coefficient and $F_{a p}$ is the applied force. [21].

Fig. 2. "Hard dead zone" 


\section{Parameter Estimation}

To estimate or identify the parameters of the Maxon PMDC motor the Strejc method was applied, [24]. That is, by analyzing the step responses, for various input voltages $v(t)$, of the mechanical and electrical subsystems (3) and (6), at rotors speeds $\omega(t) \neq 0$. This allows to avoid the effects of the dead zone and Coulomb friction. Although, manufacturer's parameters data is available, table I, only constant motor $k_{m}$ was used as there is not information on the physical characteristics of the rotor. It should be noted that using manufacturer data render a model whose responses do not match actual motor responses. Therefore, it can be concluded that motors conditions of operations affect parameters estimation. That is, manufacture procedure for parameters measurement not necessarily are those required for control purposes.

Table 1. Maxon Motor Data

\begin{tabular}{|c|c|}
\hline Parameter & Value \\
\hline$R$ & $10.6 \Omega$ \\
\hline$L$ & $0.825 \times 10^{-3} \mathrm{H}$ \\
\hline$k_{m}$ & $50.2 \times 10^{-3} \mathrm{Nm} / \mathrm{A}$ \\
\hline$J$ & $12.1 \times 10^{-7} \mathrm{~kg} \mathrm{~m}$ \\
\hline
\end{tabular}

\subsection{Blocked Rotor Test}

Although the counter electromotive force was neglected the electrical subsystem was estimated with a blocked rotor to assure $E_{a}=0$ in equation (1). Therefore, resistance and inductance of the electrical subsystem can be obtained based on the principle that the PMDC motor behaves as a $R L$ circuit as shown in figure 4.

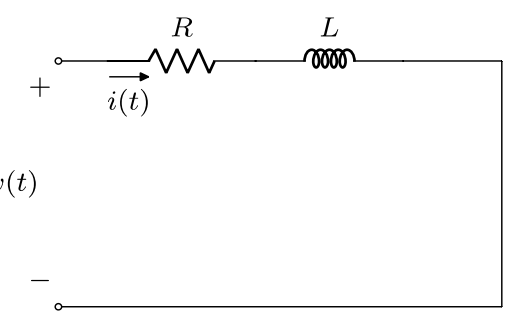

Fig. 4. RL Circuit

Current $i(t)$ is obtained from the current sensor of the DCMCT system. The gain of the transfer function (3) can be determined by transient and steady state responses of the PMDC motor current $i(t)$ when the rotor is blocked. The responses, for five different input voltages, are shown in figure 5.

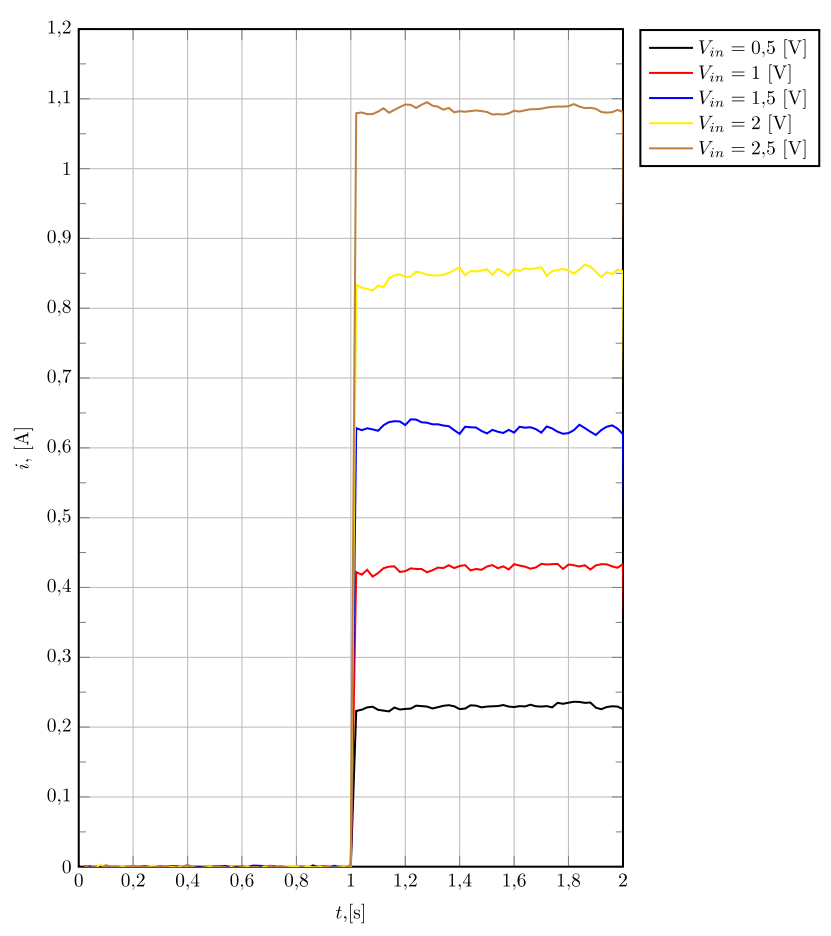

Fig. 5. Current response in the blocked rotor test.

The steady state gain obtained for these responses is $K_{e}=0.421762$ with a steady state time $t_{s}=0.03 \mathrm{~s}$. Thus, the time constant is $\tau_{e}=$ 0.0075 . The resulting transfer function for the electrical subsystem is shown in (9).

$$
G_{e}(s)=\frac{K_{e}}{\tau_{e} s+1}=\frac{0.421762}{0.0075 s+1}
$$

Therefore, by equation (3) resistance $R$ and inductance $L$ result in:

$$
\begin{gathered}
R=\frac{1}{K_{e}}=2.3724 \Omega \\
L=\tau_{e} R=17.7933 \times 10^{-3} \mathrm{H}
\end{gathered}
$$

\subsection{Speed measurement at a step input}

The mechanical parameters are estimated following the same procedure as that applied to the electrical subsystem. That is, is possible to identify equation (6) by the analysis of the transient and steady state responses of the rotor velocity $\omega(t)$ to different step input voltages.

Rotor velocity $\omega(t)$ responses are shown in figure 6 . 


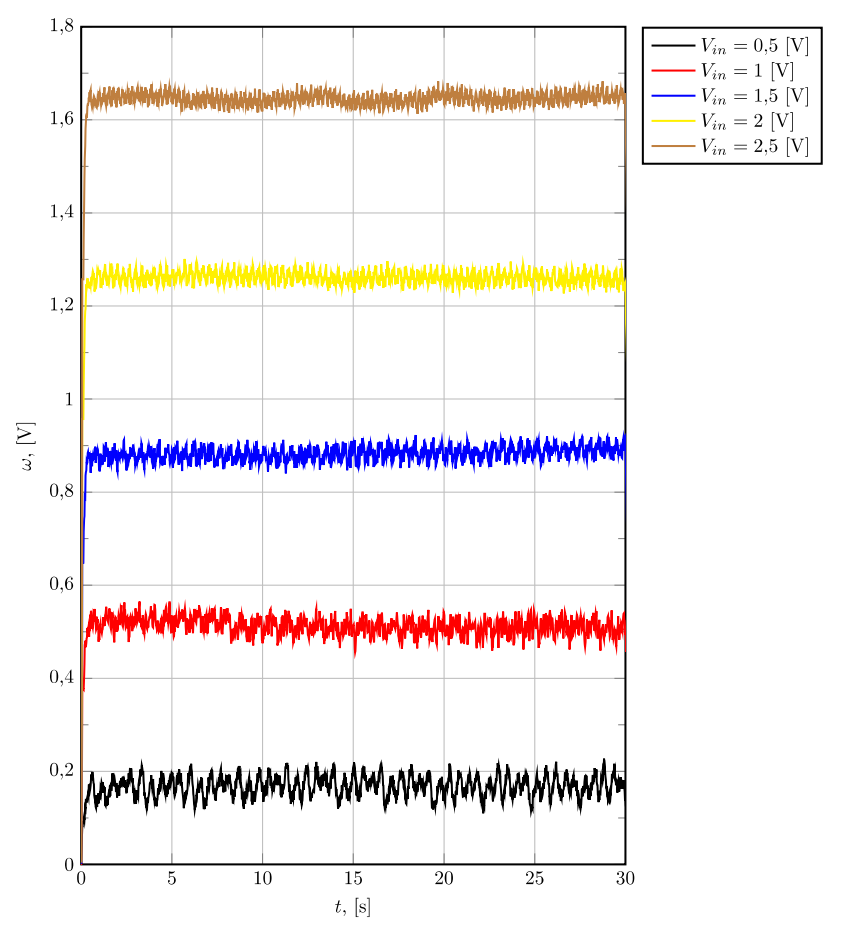

Fig. 6. Speed response at a step input.

From figure 6, steady state gain and establishment time result in $K_{m o t}=0.545287$ and $t_{s}=0.32 \mathrm{~s}$, respectively. Thus, process time constant is $\tau_{m o t}=0.08$ and the resulting motor transfer function is given by equation (12).

$$
G_{s}=\frac{0.545287}{0.08 s+1}
$$

From equation (6) the viscous friction coefficient $b$ and the inertia $J$ can be calculated, resulting in:

$$
\begin{gathered}
b=\frac{k_{m}}{K_{m o t} R}=0.0388 \mathrm{~N} \mathrm{~m} / \mathrm{rad} / \mathrm{s} \\
J=\tau_{m o t} b=3.10442 \times 10^{-3} \mathrm{~kg} \mathrm{~m}^{2}
\end{gathered}
$$

It is well known that viscous friction coefficient $b$ depends on speed, however, it will be assumed constant since the objective is to obtain and validate a motor model for a narrow speed range around zero speed.

\subsection{Nonlinear parameters}

Coulomb friction is estimated by experimental observation. That is, the Coulomb friction value is manually adjusted based on the model's speed and position responses to a triangular input voltage signal and compared to actual motor responses. Similarly, the viscous friction value is adjusted so that the model responses are as similar as possible to the actual motor responses. The triangular input signal allows better observation of this non-linear phenomena when the system operates at different speeds around zero speed.

The values for the Coulomb coefficient and the viscous coefficient obtained are $b_{c}=0.005 \mathrm{Nm} /$ $\mathrm{rad} / \mathrm{s}$ and $b_{v}=0.0314 \mathrm{Nm}$, respectively.

The dead zone parameters are estimated by measuring the input voltage and the current generated in the armature at which the motor starts to move, so the magnetic torque breaking point of the dead zone can be estimated, equation (4).

The motor starts to move at $\pm 0.3 \mathrm{~V}$ with an armature current $i(t)=0.1265 \mathrm{~A}$. Therefore, the torque break points are $T_{m \delta_{r}}= \pm 6.35 \times 10^{-3} \mathrm{Nm}$.

Finally, the estimated parameters of Maxon PMDC motor of the DCMCT system are shown in the table 2.

Table 2. Parameters Estimated by Experimental

\begin{tabular}{|c|c|}
\hline \multicolumn{1}{c}{ Parameter } & Value \\
\hline$R$ & $2.3724 \Omega$ \\
\hline$L$ & $17.7933 \times 10^{-3} \mathrm{H}$ \\
\hline$k_{m}$ & $50.2 \times 10^{-3} \mathrm{Nm} / \mathrm{A}$ \\
\hline$J$ & $3.10442 \times 10^{-3} \mathrm{~kg} \mathrm{~m}{ }^{2}$ \\
\hline$b_{v}$ & $0.0314 \mathrm{~N} \mathrm{~m} / \mathrm{rad} / \mathrm{s}$ \\
\hline$b_{c}$ & $0.005 \mathrm{~N} \mathrm{~m}$ \\
\hline$\delta_{v}$ & $6.35 \times 10^{-3}$ \\
\hline
\end{tabular}

\section{Model MATLAB®/SIMULINK ${ }^{\text {TM }}$}

in

The Matlab ${ }^{\circledR} /$ Simulink $^{\mathrm{TM}}$ model of the PMDC motor is shown in figure 7 , where the dead zone is located between the electrical and mechanical subsystems.

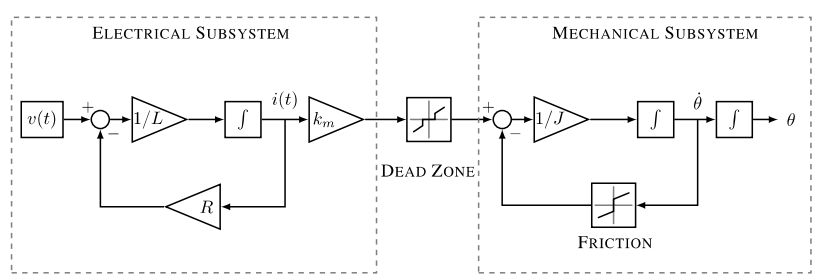

Fig. 7. Matlab ${ }^{\circledR} /$ Simulink ${ }^{\mathrm{TM}}$ PMDC motor model.

The symmetric dead zone is modeled by Matlab ${ }^{\circledR} /$ Simulink $^{\mathrm{TM}}$ blocks as shown in figure 8 . The $k_{m} i(t)_{i n}$ represents the magnetic torque generated by the electric subsystem and the 
$k_{m} i(t)_{\text {out }}$ is the magnetic torque supplied to the mechanical subsystem. For values less than $T_{m \delta_{r}}=$ $\pm 6.35 \times 10^{-3} \mathrm{Nm}$ no torque is induced to the mechanical subsystem.

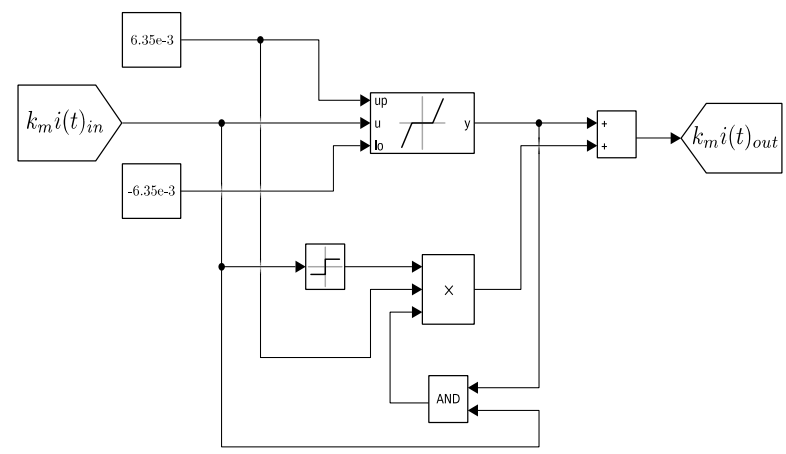

Fig. 8. Hard dead zone Matlab ${ }^{\circledR} /$ Simulink $^{\mathrm{TM}}$ model.

Friction force is modeled using the Matlab ${ }^{\circledR} /$ Simulink $^{\mathrm{TM}}$ function block. The code for the friction function is shown in figure 9.

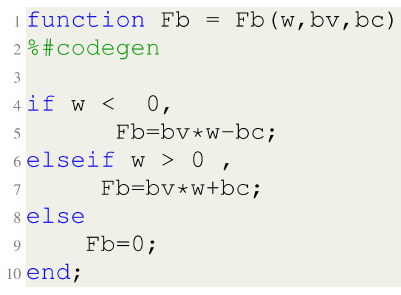

Fig. 9. Matlab code for the friction force function.

\section{Validation}

The non-linear PMDC motor model and the real system are compared using the Matlab $® /$ Simulink $^{\mathrm{TM}}$ real time package. For the tests, a triangular input is supply for both, the model, and the real system, in the voltage range $[-2,2] \mathrm{V}$. Through this test the speed and position response are observed.

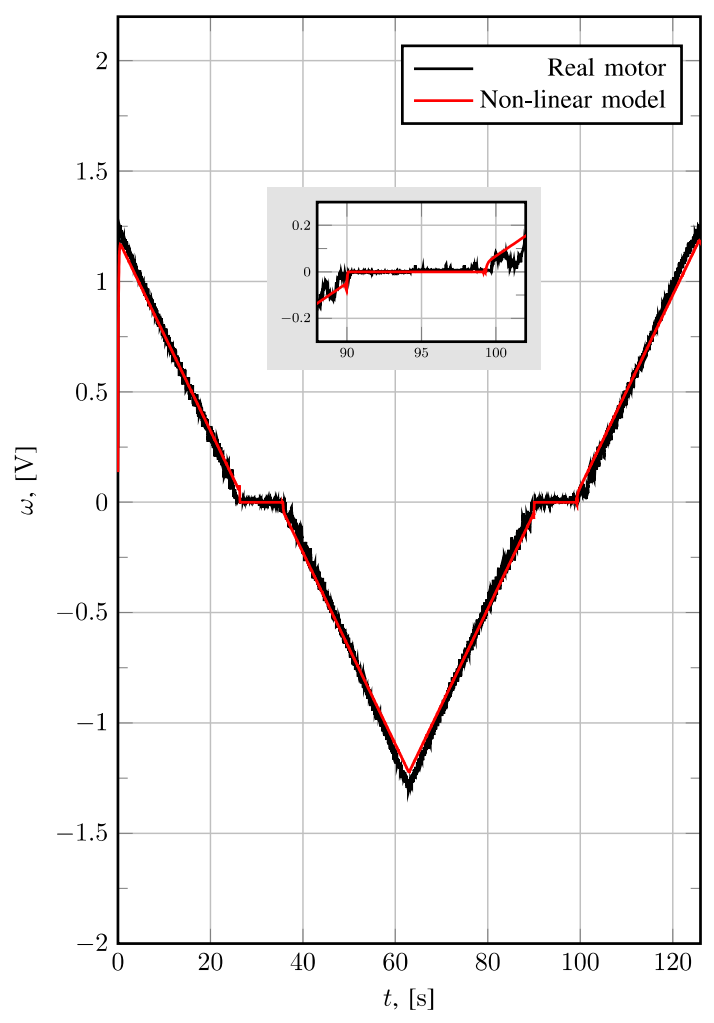

Fig. 10. Speed comparison.

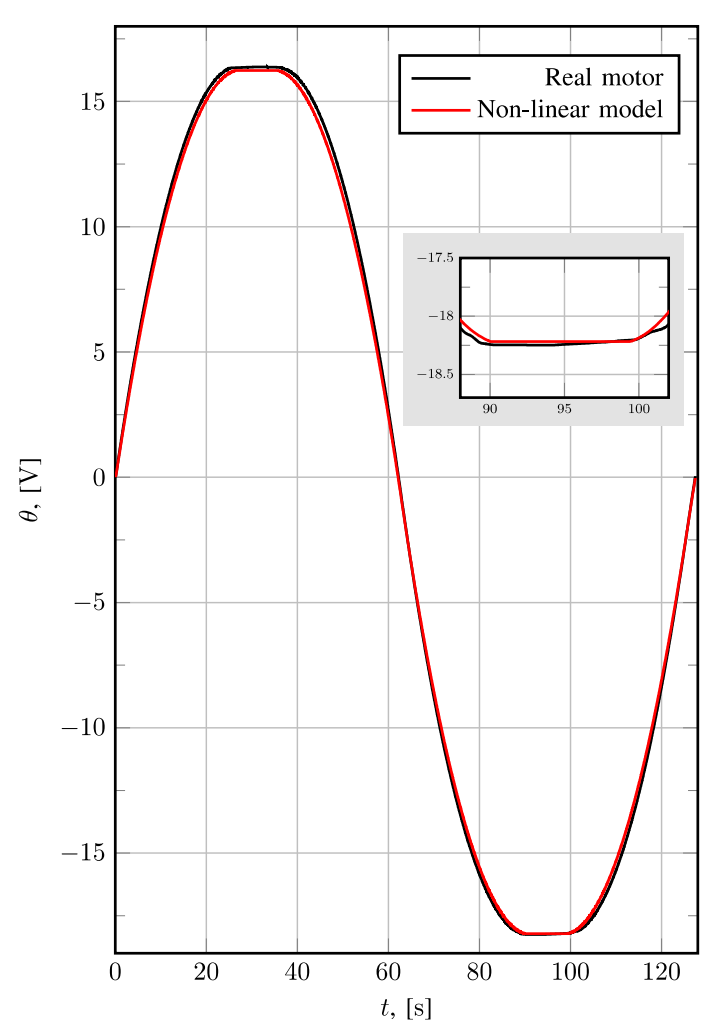

Fig. 11. Position comparison.

Rotor speed values are obtained by a tachometer including in the DCMCT system, which provides a 
scaled signal in a range of $\pm 5 \mathrm{~V}$, where $1 \mathrm{~V} \approx 74 \mathrm{rad} / \mathrm{s}$. Speed response is shown in figure 10 where it is observed that the model response is very similar to the PMDC Maxon motor response, especially at low speeds. Similarly, the model can accurately represent the phenomenon of the dead zone with a very small error rate. For high speeds, the error tends to increase, this is because the viscous friction coefficient was assumed constant. Modeling and identifying viscous friction as a function of speed will render a motors model more suitable for wider speed ranges.

Rotor position responses are shown in figure 11. These responses where obtained by integrating the tachometer signal output and comparing it with the response of the non-linear model. The dead zone can be clearly seen at the top and bottom of the curve.

Although error is not statistically validated by its absolute mean and standard deviation, is possible to notice that the error between both responses is small for the tested voltage range.

\section{Polynomial model of friction}

Observing the responses in section 5, for high speeds values, the error increase between the model and the response of the real motor, because the coefficient of viscous friction is considered constant when it is known that in reality this term varies with speed. For this reason, a new friction model is proposed and obtained by the response of the motor to different input voltages, using a polynomial approximation.

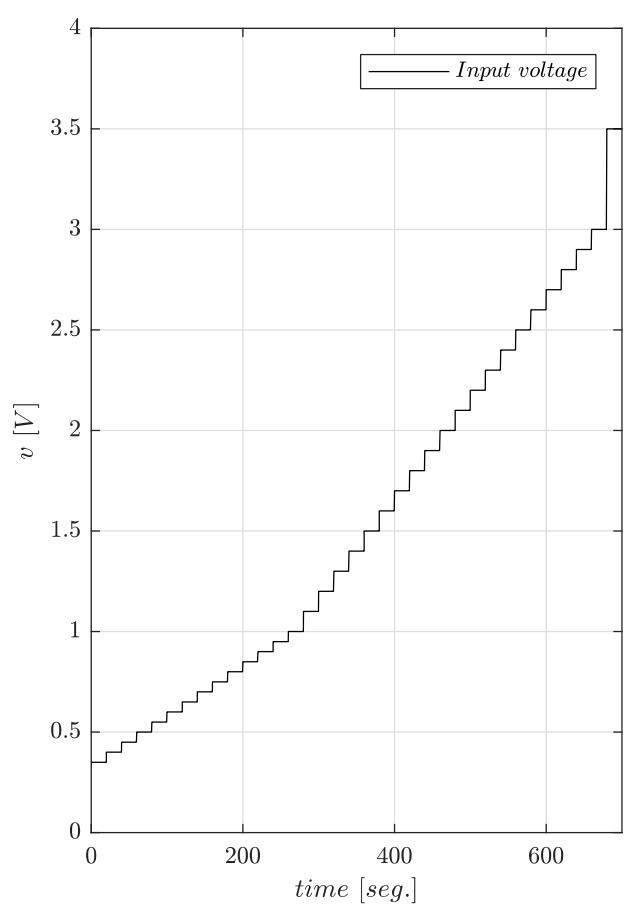

Fig. 12. Input voltage for polynomial friction aproximation.

The input voltage signal is shown in figure 12 and the speed response to this input is observed in figure 13.

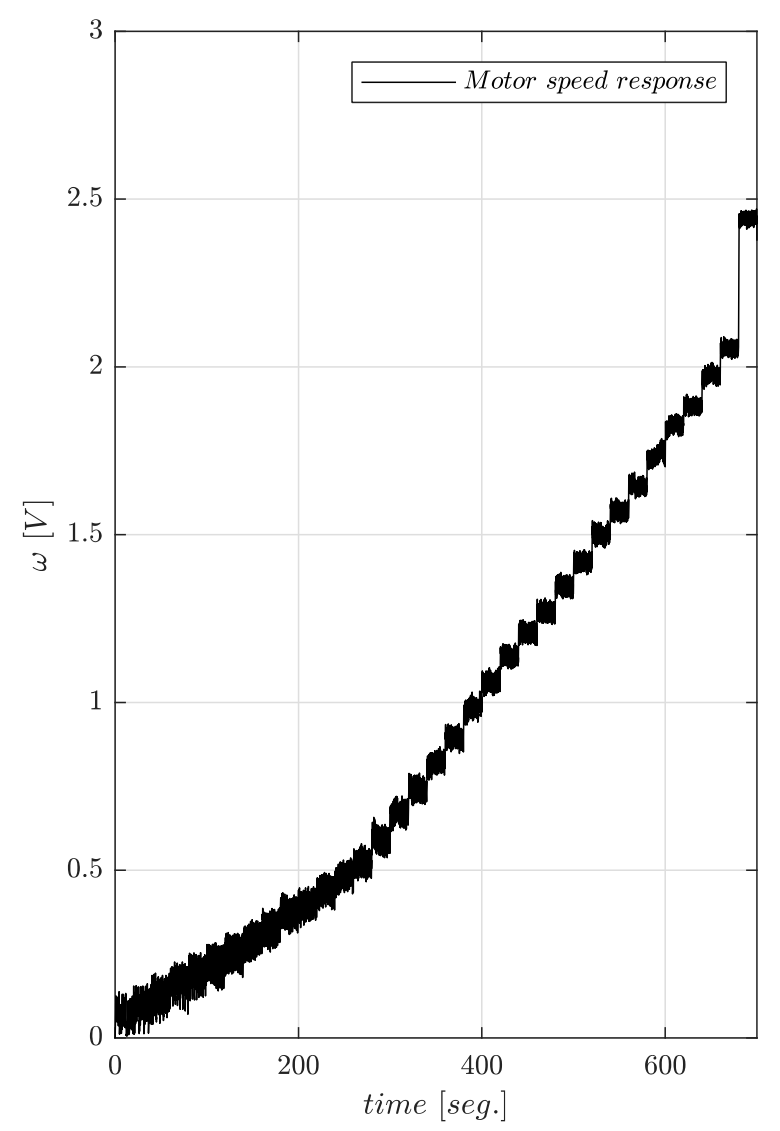

Fig. 13. Motor speed response. 
For each input voltage a viscous friction coefficient value is determined following the same procedure of Section 3.2, obtaining a set of data corresponding to viscous friction versus rotor speed that allows to model the phenomenon through a polynomial approximation as shown in figure 14 .

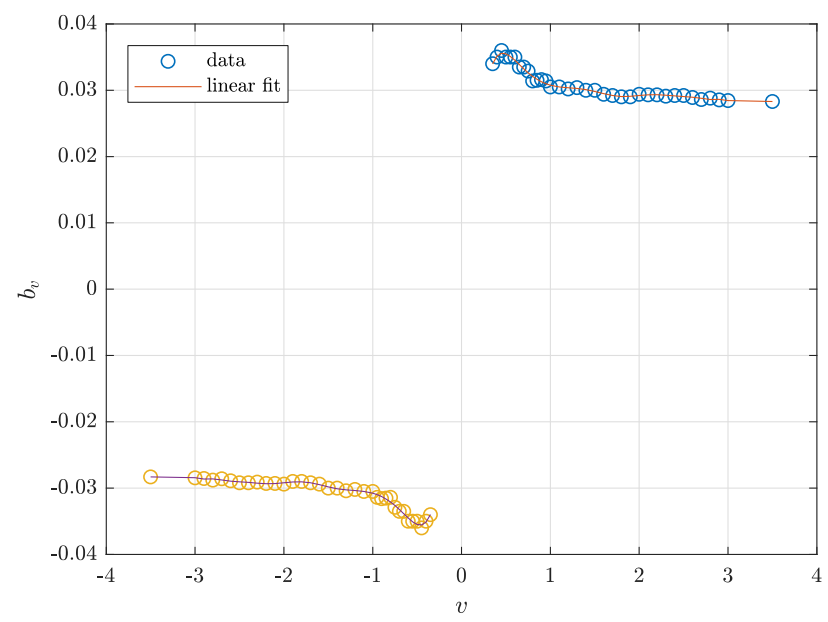

Fig. 14. Polynomial aproximation of friction.

Each circle in Figure 14 represents the value of the viscous friction coefficient obtained at a certain input voltage, while the polynomial fit of all the obtained viscous friction values is represented by the solid line. Therefore, the friction model used in the nonlinear model of the PMDC motor is given by:

$$
F_{b}=P(\omega) \omega \pm b_{c}
$$

where $P(\omega)$ represents the polynomial obtained from the viscous friction values. For this representation of friction, both the polynomial obtained and the Coulomb coefficient, $b_{c}$, are adjusted manually to obtain a speed response like that of the real motor.

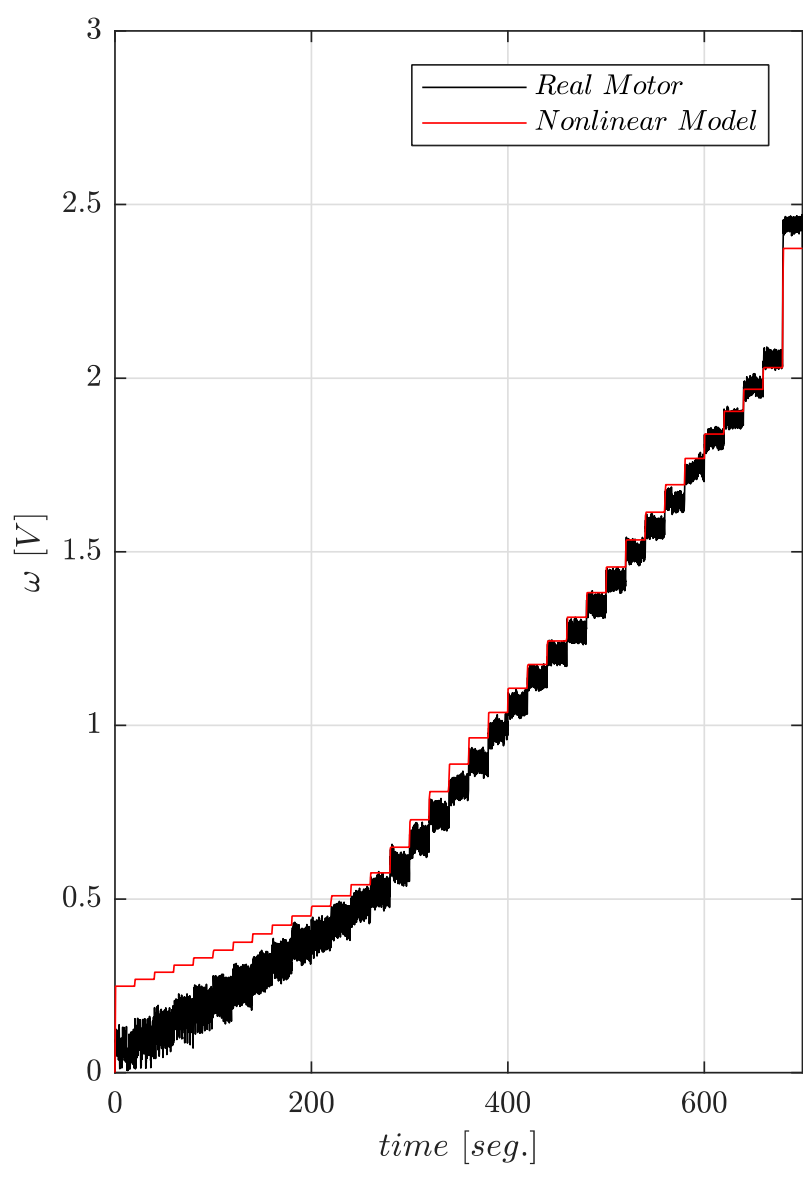

Fig. 15. Comparison of the speed response between the real motor and the nonlinear model with the polynomial friction model.

Comparison of the speed responses between the real motor and the non-linear model with the polynomial friction approximation is shown in figure 15 . As can be seen in the figure, for relatively high speeds the response of the non-linear model is very similar to the response of the real system, however at low speeds the error between both responses is greater. Therefore, we can determine that this model is a good approximation for the implementation of controllers designed for speed control, since it automatically adjusts the friction values of the system.

\section{Conclusion}

In this work, a non-linear PMDC motor model is proposed. This model takes into account two of the most important nonlinear phenomena, dead zone and friction, that significantly affect the performance of designed controllers, especially in those systems operating at low speeds or for those in whose high precision position control is essential. 
The dead zone is modeled using an approach called the "hard dead zone", where the inertia breakdown is represented as a sudden displacement, better describing the phenomenon present in the real system. The friction phenomenon is represented using the Coulomb plus viscous friction model which better represent the friction a low and zero speed.

To obtain the model, the motor parameters are estimated using the transfer functions corresponding to the electrical and mechanical subsystem by applying the Strecj method.

The model was validated with the Quanser DCMCT system that has an integrated Maxon PMDC motor. The non-linear PMDC motor model was simulated and compared with the real system for a certain range of voltages where it was possible to clearly observe the non-linear phenomena present in the analyzed motor, observing through the responses of both the linear model and the motor Maxon DC permanent magnet, that the model obtained is a very good approximation of the real system at low speeds. Finally, to obtain a PMDC motor model that represents the system in a wider range of speeds, a new friction model was proposed, modeling viscous friction, which is the value of friction that varies with the speed of the system; using a polynomial approximation. The non-linear model using this friction model provides good results at relatively high speeds, contrary to what is obtained by considering viscous friction as a constant. Therefore, it is possible to conclude that for the development and implementation of position controllers, using the non-linear model that considers the viscous friction value as a constant is an excellent option; however for controllers designed for PMDC motor speed control, using the model that automatically adjusts the viscous friction value may provide better results.

\section{References:}

[1] N. P. Mahajan and S. Deshpande, "Study of nonlinear behavior of dc motor using modeling and simulation," International Journal of Scientific and Research Publications, vol. 3, no. 3, pp. 576-580, 2013.

[2] S. Syukriyadin, S. Syahrizal, G. Mansur, and H. Ramadhan, "Permanent magnet dc motor control by using arduino and motor drive module bts7960," in IOP Conference Series: Materials Science and Engineering, vol. 352, no. 1. IOP Publishing, 2018, p. 012023.

[3] J. E. Carryer, R. M. Ohline, and T. W. Kenny, Introduction to mechatronic design, 2011.
[4] A. Hughes and B. Drury, Electric motors, and drives: fundamentals, types and applications. Newnes, 2019.

[5] S. Moussavi, M. Alasvandi, and S. Javadi, "Speed control of permanent magnet dc motor by using combination of adaptive controller and fuzzy controller," International Journal of Computer Applications, vol. 52, no. 20, 2012.

[6] A. Alkamachi, "Permanent magnet dc motor (pmdc) model identification and controller design," Journal of Electrical Engineering, vol. 70, no. 4, pp. 303-309, 2019.

[7] H. Chu, W. Tao, B. Gao, Q. Liu, and H. Chen, "Speed control of the permanent-magnet dc motor subjected to uncertainty and disturbance," in 2016 35th Chinese Control Conference (CCC). IEEE, 2016, pp.46644669.

[8] S. Brennan and A. Alleyne, "Dead-zone nonlinearities," 1999.

[9] J.-H. Horng, "Neural adaptive tracking control of a dc motor," Information sciences, vol. 118, no. 1-4, pp. 1-13, 1999.

[10] J. d. J. Rubio, Z. Zamudio, J. Pacheco, and D. Mujica Vargas, "Proportional derivative control with inverse dead-zone for pendulum systems," Mathematical Problems in Engineering, vol. 2013, 2013.

[11] C.-K. Lai, "A hybrid scheme motion controller by sliding mode and two degree-of-freedom controls to minimize the chattering," Mathematical Problems in Engineering, vol. 2014, 2014.

[12] C.-C. Chiang, "Adaptive fuzzy tracking control for uncertain nonlinear time-delay systems with unknown dead-zone input," Mathematical Problems in Engineering, vol. 2013, 2013.

[13] Mercorelli, P. "Parameters identification in a permanent magnet three-phase synchronous motor of a city-bus for an intelligent drive assistant". International Journal of Modelling, Identification and Control, 21 (4), pp. 352-361. 2014

[14] Khaburi, D.A., Shahnazari, M. "Parameters identification of permanent magnet synchronous machine in vector control". The 10th European Conference on Power Electronics and Applications, EPE 2003, 2-4 September, Toulouse, France, 2003

[15] J. D. Fortgang, L. E. George, and W. J. Book, "Practical implementation of a dead zone inverse on a hydraulic wrist," in ASME 2002 international mechanical engineering congress and exposition. American Society of 
Mechanical Engineers Digital Collection, 2002, pp. 149-155.

[16] F. MONASTERIO and A. Gutiérrez, "Modelo lineal de un motor de' corriente continua," 2012.

[17] D. Recker, P. Kokotovic, D. Rhode, and J. Winkelman, "Adaptive nonlinear control of systems containing a deadzone," in [1991] Proceedings of the 30th IEEE Conference on Decision and Control. IEEE, 1991, pp. 21112115.

[18] C. Hu, B. Yao, and Q. Wang, “Adaptive robust precision motion control of systems with unknown input dead-zones: A case study with comparative experiments," IEEE Transactions on Industrial Electronics, vol. 58, no. 6, pp. 2454-2464, 2010.

[19] T. Wescott, "Controlling motors in the presence of friction and backlash," in Wescott Design Services, Seminars, vol. 42, 2013, pp. 2-3.

[20] H. Olsson, K. J. Astrom, C. C. De Wit, M. G* afvert, and P. Lischinsky, "Friction models and friction compensation," Eur. J. Control, vol. 4, no. 3, pp. 176-195, 1998.

[21] V. Van Geffen, "A study of friction models and friction compensation," 2009.

[22] B. Armstrong-Helouvry, P. Dupont, and C. C. De Wit, "A survey of" models, analysis tools and compensation methods for the control of machines with friction," Automatica, vol. 30, no. 7, pp. 1083-1138, 1994.

[23] Y. Liu, J. Li, Z. Zhang, X. Hu, and W. Zhang, "Experimental comparison of five friction models on the same test-bed of the micro stickslip motion system," Mechanical Sciences, vol. 6, no. 1, p. 15, 2015.

[24] S. Skoczowski and A. Osadowski, "A simple identification method for the order of the strejc model and its application to autotuning," in Intelligent Components and Instruments for Control Applications 1994. Elsevier, 1994, pp. 319-325.

\section{Contribution of individual authors to the creation of a scientific article}

Arturo C. Pérez-Gómez performed the simulations, ran the experiments, and contributed in the analysis and approach strategy.
Jesús U. Liceaga-Castro and Irma I. Siller-Alcalá were the leaders and responsible of the project and contribute with the analysis and approach strategies.

\section{Sources of funding for research presented in a scientific article or scientific article itself}

The project was funded by the UAM-Azc and by CONACYT scholarship grant to Arturo C. PérezGómez.

\section{Creative Commons Attribution License 4.0 (Attribution 4.0 International, CC BY 4.0)}

This article is published under the terms of the Creative Commons Attribution License 4.0 https://creativecommons.org/licenses/by/4.0/deed.en_US 